\title{
ULTRA WIDEBAND RADAR FOR RESPIRATORY MONITORING ON SLEEP POSITION
}

\author{
Nurul Qashri Mahardika T², Erfansyah Ali², Fiky Yosef Suratman ${ }^{3}$ \\ ${ }^{1,2,3}$ School of Electrical Engineering, Telkom University \\ ${ }^{1}$ nurulqikaa@gmail.com, ${ }^{2}$ erfansyahali@telkomuniversity.ac.id, ${ }^{3}$ fiky.y.suratman@ieee.org
}

Manuscript received June 8, 2020; revised June 5, 2021; accepted June 8, 2021

\begin{abstract}
Sleep apnea is a sleep disorder that has a relation with respiratory system during sleep. One of the sleep apnea characteristic is suddenly stop breathing during sleep. People have the different of respiratory rate (RR) which is affected by sleep positions and body mass index (BMI).There are four sleep positions affecting the respiratory rate (RR). Polysomnography (PSG) is conventionally used to analysis the sleep apnea. This technique requires body contact that might be uncomfortable for the patient. In this study, the Xethru X4M200 radar sensor is proposed as non-contact tool to detect the RR by implementing the Doppler effect. Furthermore, the relation between RR with the sleep position and the BMI are discused. For that purpose, 20 participants (10 males and 10 females) with different BMIs and sleep positions are examined by monitoring their chest movement. This method is able to detect the indication of bradypnoea or tachypnoea. Futher systematic study and more participants are required to confirm our results and provide better non-contact technique for RR measurement.
\end{abstract}

Keywords: Respiration monitoring, doppler, respiration per minute, body mass index.

DOI: $10.25124 /$ jmecs.v8i1.2873

\section{Introduction}

Sleep is an important for life and health. A study by Khan et al. [1] defined four sleep positions namely, left, right, supine, and freefall which make different effects on heart. The study also recommended the oblique side sleeping position. In this study, the cardiac output was found to be higher in supine position than another position. However, in several cases, the blood volume or cardiac output at the right or left position was also found higher than supine position.

The disruption of sleep has a profound effect on uncomfortable when waking up, one cause of sleep disruption is in part respiratory system known as sleep apnea. Normal breathing range for adults is $12-20$ respiration per minute(RPM) [1]. One of characteristic sleep apnea is breathing shallow suddenly stops for a moment during sleep.[2]. That causes blood pressure, stroke, fail- ure heart, heart attack, diabetes, and depression $[2]$.

In medical study, sleep apnea research uses direct contact methods. For example is the polysomnogram (PSG) which monitors many body functions such as electroencephalogram, electrooculography, electromyogram, and electrocardiogram to monitor respiratory airflow and peripheral pulse oximetry during sleep [3]. Unfortunately, the use of PSG that attaches electrodes to the body creates discomfort and limitations on movement.

Another method is to use the non-contact method such as radar. The radar which relies on the doppler effect to improve target detection is called a doppler radar [4]. This type of radar has been used for many applications including small movement detection such as breath activity. Hence, this Doppler radar should be suitable for detecting chest movement caused by lung volume 
change upon breathing $[4,5]$.

To detect small movement requires radar with high resolution [6]. study conducted by Ref.[7] showed that sleep monitoring using doppler radar was more effective than PSG. Data collection which was carried out on these researcher targets were 25-28 years old with height around 180 $-185 \mathrm{~cm}$ and body weight around $80-100 \mathrm{~kg}$. A research conducted by Cristian in 2016 [8] used radar with frequency $24 \mathrm{GHz}$. The results showed that $24 \mathrm{GHz}$ radar achieves accuracy more than 90\% compared to PSG. Furthermore, this study also found that sleep disorders in the respiratory system were more common in males compared to females.

In this study, Xethru X4M200 radar is used to detect RR of 20 participants (10 males and 10 females) who have various BMI. Four sleep positions are varied as explained in Ref [1]. Our results shows that the chest movement can be monitored using this method. However, more systematic study with different BMI and various sleep disorders should be conducted in order to obtain more accurate connection between the RR, sleep position, and BMI.

\section{Respiration Detection}

\subsection{Xethru X4M200}

The sensor used in this study is the X4M200 sensor to detect vital sign data such as respiration rate, movement target, and breathing pattern. $\mathrm{XeThru} \mathrm{X} 4$ is an ultra-wideband impulse-radio radar system (IR-UWB) and using pulse-Doppler processing. Fig 1 is XeThru module X4M200. It is a module with respiration sensor which has an ability to monitoring sleep position and rpm target. In addition, the module is also capable of tracking movement and flexible time. XeThru X4M200 works with a frequency of 7,290 GHz. In XeThru X4M200, the radar transmits electromagnetic waves over the transmission antenna. The electromagnetic wave is subsequently reflected to the object, and after that, the waveform is reflected and received on the receiver antenna.

Xethru X4M200 specification shows on Table 1. Using an oscillator and a transmission antenna, the coherent signals are emitted at a coherent tone $(27 \mathrm{MHz})$. The distance between the radar and the object was determined by pulse interval. Since the pulse interval is the available time for the pulse to reach the distancing point, the quiet interval between those pulses has a direct impact on the range of the radars. Data generated by the XeThru X4M200 target detector shows as Fig 2.
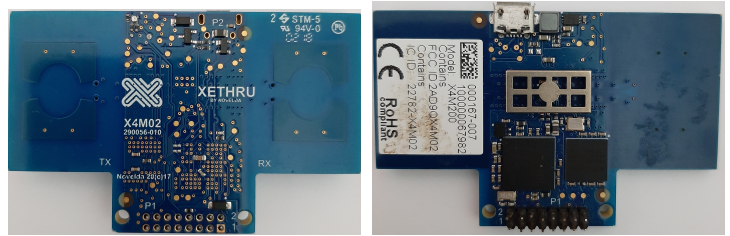

Fig. 1. XeThru X4M200

Table 1: Xethru X4M200 Spesification [9]

\begin{tabular}{|c|c|}
\hline Specification & Value \\
\hline Wave & CW \\
\hline \multirow{2}{*}{ Frequency } & Low $: 6.0-8.5 \mathrm{GHz}$ \\
& High $: 7.25-10.20 \mathrm{GHz}$ \\
\hline Range & $0.40 \mathrm{~m}-5.00 \mathrm{~m}$ \\
\hline Unit sensitivity & Minimum $: 0$ \\
& Maximum $: 9$ \\
\hline
\end{tabular}

\subsection{Radar Signal System}

There are two types of waves on the radar system, which are continuous wave $(\mathrm{CW})$ and pulse radar [9]. In this research using $\mathrm{CW}$ in radar system. In case of moving target detection, CW radar continuously transmit radio frequency waves are transmitted and received at the same time that moving target. The movement caused by the small displacement of the chest movement of the target can be analyzed as the shifting of certain phases of the reflected waves [7]. From that reason, the relation between radar doppler and doppler frequency referred to doppler frequency can be calculated using Eq (1), where $D$ is doppler frequency, $v$ for radial velocity, and $c$ is the speed of light in free space. When the target moves towards the reference point, $v$ will be positive, resulting in a positive $D$ in the obtained signal, and vice versa when the target moves away from the reference point [9]. As a target moves towards the radar device, it induces a frequency change in the transmitted and received signals, which is used to calculate the target's velocity.

$$
D=f_{r x}-f_{t x}=\frac{2 v f_{t}}{c}
$$

\subsection{CW Radar System for Respiration}

Small chest movement caused cardiac activity which manifests as the echo signals emitted by the sensor to the target using doppler effect principle. The electromagnetic signal emitted to target chest and echo signal reflected from target generate a phase change called as doppler shift [10]. The doppler shift generates RPM for every respiration, inhale and exhale. The RPM graph caused by doppler effect is shown in Fig.2 


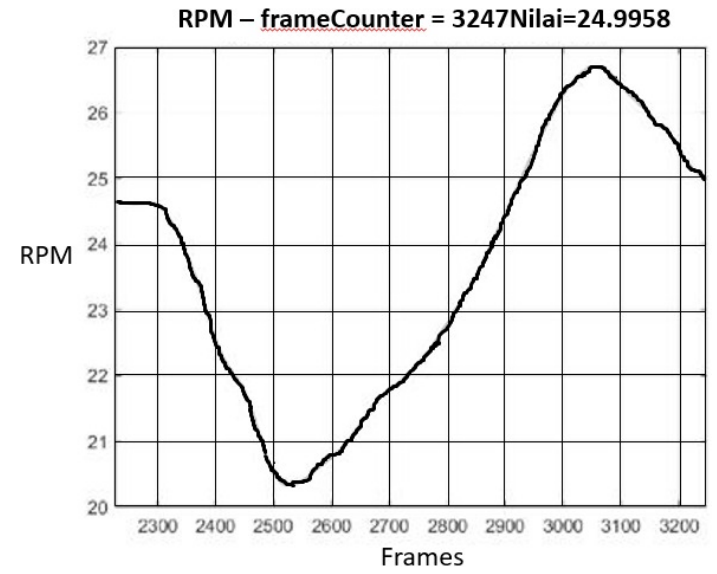

Fig. 2. Xethru X4M200 detect and lock target

The relationship between the frequency shift is called the doppler effect, so that the speed of the target using Eq (1). Target towards the radar system causes a frequency shift. The relation between frequency shift is doppler radar reffered radar system and the radial velocity $(\mathrm{v})$ the target. In general, as shown in Fig. 3 breathing patterns have a relationship with breathing activity, which can be identified from small chest movement. $\mathrm{Tt}$ is a wave of signal emitted, $\mathrm{Rt}$ is a wave reflected by the target against the sensor, $\mathrm{d}+\mathrm{x}(\mathrm{t})$ is the distance between the object and the signal source [7]. Antenna transmitted electromagnetics signal reflected and received signal target to detect chest movement as known in Eq (2-3), where $\lambda$ and $\mathrm{d}$ are the distance between the target and the radar and wavelength of the transmitted signal respectively and f and $A_{T}$ represent the frequency and amplitude of the transmitted signal. A signal reflected by the target (the doppler signal) is received and modulated by the receiver in equation $(2)$, where the and values are as frequencies that transmit the signals and amplitude.

$$
\begin{gathered}
S_{T}=A_{T} \cos (2 \pi f t) \\
S_{R}=A_{R} \cos \left(2 \pi f t+\frac{4 \pi}{\lambda} d\right)
\end{gathered}
$$

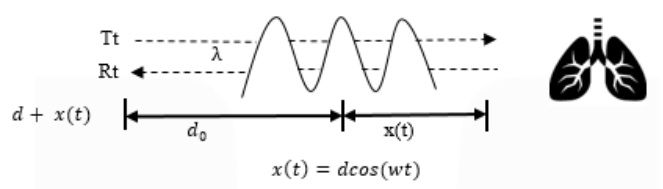

Fig. 3. Breathing pattern relationship between inhale and exhale

\subsection{Respiratory rate and Body Mass Index (BMI)}

Lungs are the respiratory organ, as part of the respiration circulatory. Their function is to exchange oxygen from the air with carbon dioxide from blood. This condition called as breathing. If heart or lungs do not work properly, the health respiration of the human body is also affected. Respiratory rate during sleep is one of the significant vital signs. RR change during can be used to determine sleep quality and also to predict some sleep disease. Respiration during sleep is different from wakefulness condition. The RPM detection during sleep can be used to detect and differentiate between various respiratory disorders during sleep [4]. For adult,the standar of respiration rate is described on Table 2 [10].

Table 2: Respiration Rate [10]

\begin{tabular}{|c|c|}
\hline RR & Range $($ RPM) \\
\hline Eupnoea (normal relaxed) & $12-20$ \\
\hline Normal range $>65$ years & $12-25$ \\
\hline Normal range $>80$ years & $10-30$ \\
\hline Bradypnoea (slow RR) & $<12$ \\
\hline Tachypnoea (fast RR) & $>20$ \\
\hline
\end{tabular}

A study conducted by [11] suggested that changes in sleep position are an alternative to sleep apnea therapy. In addition, BMI and changes in sleep position have an impact on the RPM results, this is related to the accumulation of fat in the anatomy of breathing. In Ref. [12], BMI is divided into 3 groups, namely normal BMI has a value less than $24 \mathrm{~kg} / \mathrm{m}^{2}$, mild obesity with a BMI value of $24.0-26.4 \mathrm{~kg} / \mathrm{m}^{2}$, and obesity with a BMI value of more than $26.4 \mathrm{~kg} / \mathrm{m}^{2}$. The study also showed that people with high obesity have worse oxygen saturation.

Table 3: Male Data Target

\begin{tabular}{|c|c|c|c|}
\hline Male & Age(old) & Weight $(\mathrm{kg})$ & Height $(\mathrm{cm})$ \\
\hline 1 & 22 & 62 & 170 \\
\hline 2 & 20 & 65 & 168 \\
\hline 3 & 22 & 51 & 168 \\
\hline 4 & 21 & 49 & 160 \\
\hline 5 & 20 & 63 & 170 \\
\hline 6 & 20 & 68 & 175 \\
\hline 7 & 21 & 74 & 175 \\
\hline 8 & 23 & 48 & 173 \\
\hline 9 & 21 & 50 & 170 \\
\hline 10 & 21 & 89 & 181 \\
\hline
\end{tabular}


Mahardika et al / Journal of Measurement, Electronics and Communication Systems

Table 4: Female Data Target

\begin{tabular}{|c|c|c|c|}
\hline Female & Age(old) & Weight $(\mathrm{kg})$ & Height $(\mathrm{cm})$ \\
\hline 1 & 21 & 53 & 154 \\
\hline 2 & 21 & 50 & 150 \\
\hline 3 & 21 & 60 & 153 \\
\hline 4 & 21 & 50 & 158 \\
\hline 5 & 21 & 40 & 154 \\
\hline 6 & 21 & 46 & 150 \\
\hline 7 & 21 & 40 & 157 \\
\hline 8 & 21 & 44 & 154 \\
\hline 9 & 22 & 70 & 15 \\
\hline 10 & 21 & 48 & 167 \\
\hline
\end{tabular}

Table 5: BMI Target

\begin{tabular}{|c|c|}
\hline Male & BMI $(\mathrm{Kg} / \mathrm{m} 2)$ \\
\hline 1 & 21.5 \\
\hline 2 & 23 \\
\hline 3 & 18.1 \\
\hline 4 & 19.1 \\
\hline 5 & 21.8 \\
\hline 6 & 22.2 \\
\hline 7 & 24.2 \\
\hline 8 & 16 \\
\hline 9 & 17.3 \\
\hline 10 & 27.2 \\
\hline
\end{tabular}

\begin{tabular}{|c|c|}
\hline Female & BMI $(\mathrm{Kg} / \mathrm{m} 2)$ \\
\hline 1 & 22.3 \\
\hline 2 & 22.2 \\
\hline 3 & 25.6 \\
\hline 4 & 20 \\
\hline 5 & 16.9 \\
\hline 6 & 20.4 \\
\hline 7 & 16.2 \\
\hline 8 & 18.6 \\
\hline 9 & 24.8 \\
\hline 10 & 17.2 \\
\hline
\end{tabular}

\section{Research Method}

\subsection{Respiration Tracking}

This study probes and detects the RPM value for each target sleep position. Matlab was used to process the RPM data. This study does not reported the radar signal processing since XeThru X4M200 already used on Xethru Explorer. The XeThru X4M200 respiration tracking steps are scematically shown in Fig. 4. The first step was initialization. This state is indicated by a red, yellow, and green light emitting diode (LED). The initialization stage carried out for 120 seconds. The purpose of this stage was to turn on the oscillator to produce optimal waves. The next stage was no movement. The sensor can not detect any movement at this stage because there was no target. If there was a target it would be changed to the next. The next step was the movement stage. The 0.5 seconds of each green and blue light emitting diode (LED) on sensors indicate that the sensor has locked the target. The last stage was breathing. The sensor was already locked the target in stable state and can stably detect chest movements as well as generate the RPM.

\subsection{Target Position}

This research conducted breathing analysis on sleep position using UWB radar. The distance

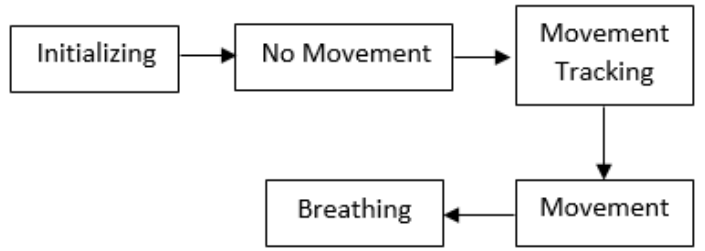

Fig. 4. Xethru X4M200 Respiration Tracking

from the sensor to the target is $1 \mathrm{~m}$ with a height of $75 \mathrm{~cm}$. This study observed RPM target based on criteria on the Table 2 with various sleep position and BMI target. The targets perform 4 sleep scenarios, namely right, left, supine, and freefall positions as presented in Fig 5 . The targets were 20 participants of which 10 are male and 10 female with an age range of 21-23 years as explained in Table 3 and 4 . The participants had healthy respiration, no chronic disease disturbance, and random BMI as presented in Table 5.

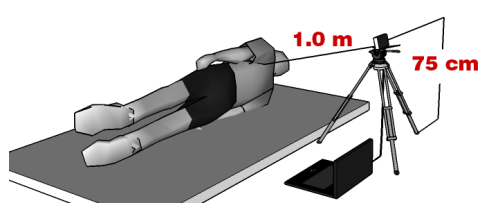

(a)

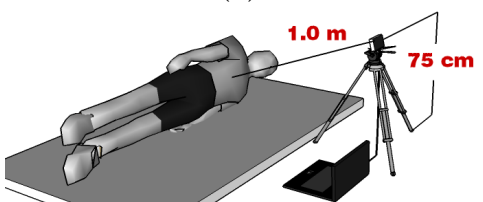

(b)

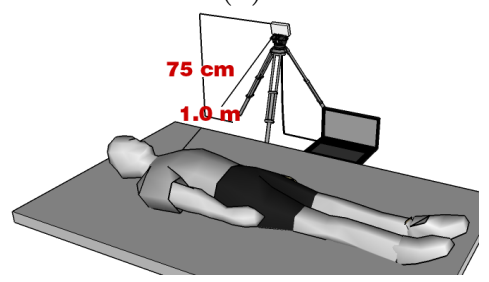

(c)

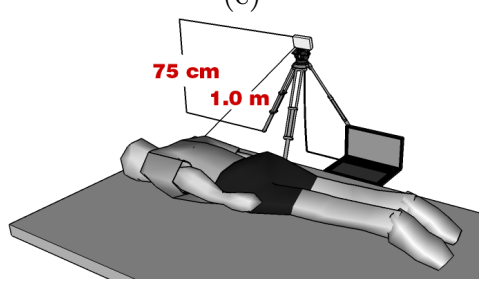

(d)

Fig. 5. Pictorial view of XeThru X4M200 and four sleeping positions of (a) right position, (b) left position, (c) supine position, and (d) freefall position 


\section{Result and discussion}

To get stable RPM, in this study the target must be in a state of sleep comfortably which provides ideal breathing movements. The measured RPM for various sleeping positions are presented in Fig. 6,7,8 and 9 with different sleeping positions. Figure 6 shows the result of RPM in the supine position. For female target, there are 5 targets with abnormal RPM results that can be classified as tachypnoea (fast RR). Meanwhile, for male data there are 3 targets with tachypnoea results (fast RR) and 1 target with bradypnoea (slow RR) results. Other targets in this position have normal RPM. Figure 6 also shows male target 1 with normal BMI of 18.1 has the RPM tachypnoea (fast RR) results, while targets 8,9 , and 10 have normal RPM even though they have abnormal BMI.

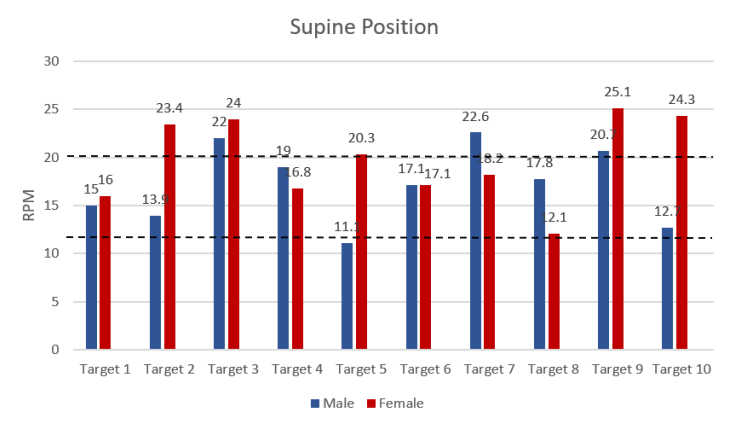

Fig. 6. RPM value for supine position

Figure 7 shows the results RPM for right position. There are 3 female targets with Tachypnoea results (fast RR) and two male targets with Tachypnoea results (fast RR). There is no clear relation between BMI and RPM. In this sleeping position, the RPM of female targets tends to increase linearly with the BMI. Three female targets with BMI of 25.6 were observed to have RPM of 25 while 5 female targets with BMI of 16.9 had the RPM of 22.9. However, 3 thin female targets with BMI around 16-27 were found to have normal RPM.

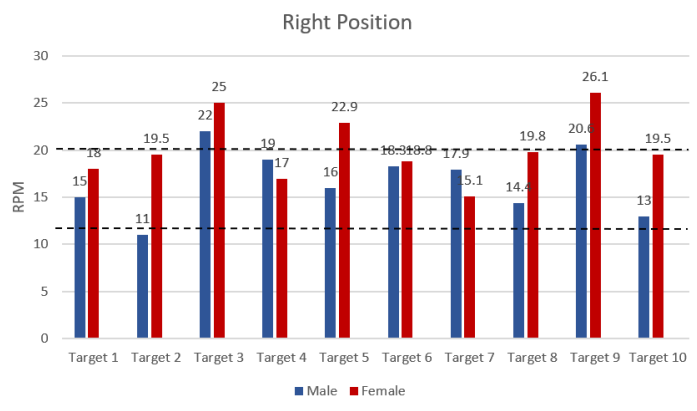

Fig. 7. RPM value for right position

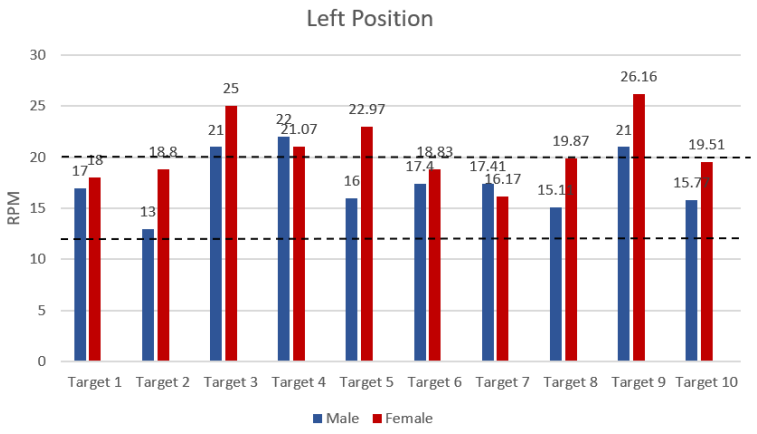

Fig. 8. RPM value for left position

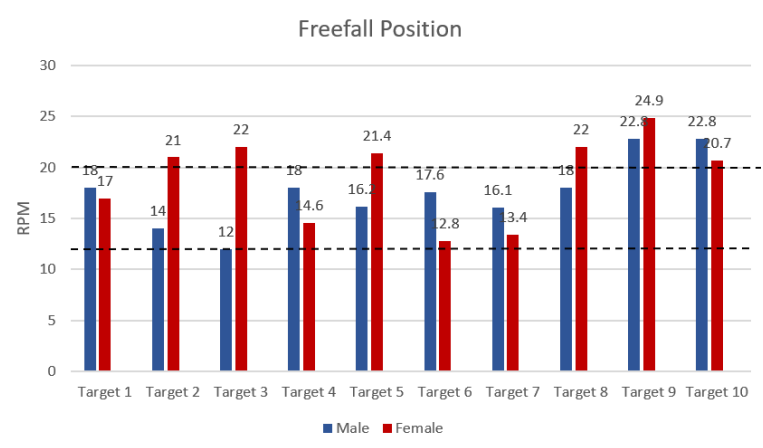

Fig. 9. RPM value for frefall position

Figure 8 shows the RPM chart of left position. There are 4 female targets with the Tachypnoea (fast RR) result. Based on table 5, fast RPM were detected on 4 females targets; 1 with BMI of 25 and 3 with normal BMI. For male targets, fast RPM was observed on 3 males with normal BMI. Figure 9 shows the target RPMs at free fall position. Six female targets had fast RPM. Three of them had mild obesities while the others had normal BMI. For male, two targets with normal BMi and obesity were found to have fast RPM.

\section{Conclusion}

We conclude that Xethru X4M200 is able to distinguish slow and fast $\mathrm{RR}$ for various sleep position and BMI. In this study, the Tachypnoea (fast RR) is found to BMI independence. The fast RR is observed in several persons either with normal or thin BMI. Furthermore, normal RPM is also observed in over weight group. Considering the sleep position, the abnormal RPM are found at 9 targets with supine position, 7 person with left position, 6 person with right position, and 8 persons with for freefall position. Despite the advantage of the Xetrhu X4M200 as non contact RPM detector, more targets and measurement should be conducted in the future in order to confirm the accuracy. 
Mahardika et al / Journal of Measurement, Electronics and Communication Systems

\section{References}

[1] Shabaz Khan, Md. Bashir Uddin, Effects of sleeping positions on cardiac output and cardiac activity analyzing blood perfusion, 2nd Intl Conf. on Electrical Engineering and Information Communicaion Technology (ICEEICT) 2015 Jahangimagar University, Dhaka1342, Bangladesh, 21-23 May 2015.

[2] Na Du, Kewen Liu, Linfei Ge, Jin Zhang, ApneaRadar : A $24 \mathrm{GHz}$ Radar-Based Contactless Sleep Apnea Detection System, in International Conference on Frontiers of Sensors Technologies, 2017.

[3] Hong Hong, Li Zhang, Chen Gu, Yusheng Li, "Noncontact Sleep Stage Estimation Using a CW Doppler Radar," In IEEE Journal on Emerging and Selected Topics in Circuit and Systems, 2017.

[4] A.A Pramudita, Fiky. Y Suratman, Dharu Arseno, and Erfansyah Ali, FMCW radar post processing method for small displacement detection, IEEE International Conference on Aerospace Electronics and Remote Sensing Technology (ICARES), 2018.

[5] Changzhi Li, Member IEEE, Victor M.Lubecke, Senior Member, IEEE, Olga Boric-Lubecke, Senior Member, IEEE, and Jenshan Lin, Fellow, IEEE, A review on recent advances in doppler radar sensors for non noncontact healthcare monitoring, IEEE Transactions on Microwave Theory and Techniques, Vol. 61, No.5, May 2013

[6] Rizky Ambarini, Aloysius Adya Pramudita,and Erfansyah Ali, Single-Tone doppler radar system for human respiratory monitoring,International Conference on Electrical Engineering, Computer Science and Informatics (EECSI), 2018

[7] H. Ming-Chun. L. J.J., X Wenyao et al., A selfcalibrating radar sensor system for measuring vital sign, in IEEE Transaction on Biomedical Circuits and System, May 2016, pp. 352-363.

[8] X4M200,X4M200 Datasheet, December 21, 2017

[9] M.Silos Viu, Somnus : An-Ultrawideband Radar-Based Approach for Neonatal Sleep State Classification, Master Thesis, Delft University of Technology, November 9,2017.

[10] Nursing Times[online],Respiratory rate 3: How to take an accurate measurement., vol.114, July 2018
[11] Kevin Bouchard, Julien Maitre, Camile Bertuglia, Sebastien Gaboury, "Activity Recognition in Smart Homes using UWB Radars". The 11th International Conference on Ambient Systems, Networks and Technologies (ANT), April 6-9, 2020

[12] Yoshiaki Itasaka, Soichiro Miyazaki, Kazuo Ishikawa, Kiyoshi Togawa, "Sleep Breathing Disorders, The Influence of Sleep Position and Obesity on Sleep Apnea," Psychiatry and Clinical Neuroscience, 2000

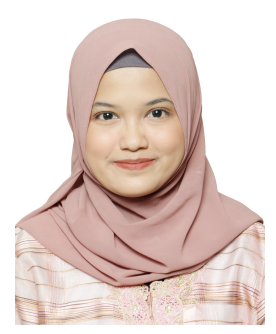

Nurul Qashri Mahardika, received her bachelor's degree major in Telecommunication Engineering, Telkom University (2020). She joined as a research assistant in Biomedical Signal Processing and Instrumentation Laboratory - Telkom University (2018-2020) and as a practicum assistant in Electronics Telecommunication Laboratory Telkom University (2019-2020). Currently, she is a master's student from Computational Medicine Laboratory - Kumoh National Institute of Technology. Her research interests are in radar, medical signal processing, and machine learning.

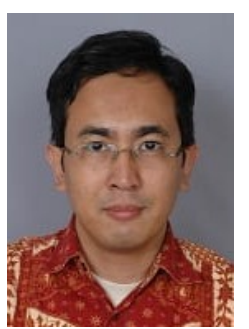

Erfansyah Ali, received his BS (2005) and MS (2012) both from Electrical Engineering Department major in Communications, Intitut Teknologi Bandung (ITB). Currently he is a lecturer at School of Electrical Engineering, Telkom University and a PhD student at National Taiwan University of Science and Technology (NTUST). His research interests are in Radar Systems, RF device and Signal Processing. 
Mahardika et al / Journal of Measurement, Electronics and Communication Systems

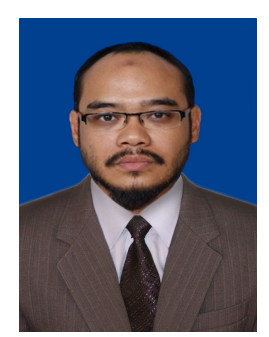

Fiky Y. Suratman (S'09 M'14) was born in Jakarta, Indonesia, in 1976. He received Bachelor Degree from Engineering Physics and Master Degree from School of Electrical and Informatics (STEI), Institut Teknologi Bandung, Bandung, Indonesia in 1998 and 2006, respectively. He was awarded scholarship from German Academic Exchange Service (DAAD) to continue his study at Technische Universitaet Darmstadt (TU-Darmstadt), Germany, until he recieved his Dr.-Ing. Degree (PhD) in 2014. Early placement in industry (Astra Microtronics Technology) from 1998 to 2001, was then followed by lectureship in Universitas Komputer Indonesia (UNIKOM). In 2007, he joined the Faculty of Electrial Engineering, Telkom University as a faculty member. He is the head of Master Degree of Electrical Engineering, Telkom University since 2014. He is a member of IEEE (Signal Processing Soiciety and Communications Society), and he currently acts as the chairman of Signal Processing Society (SPS) Indonesia Chapter. His research interest lies on Statistical Signal Processing, Radar Signal Processing, and Machine Learning. 